Supporting Information

\title{
How Tethered Probes Report the Dynamics of a Polymer near the Glass Transition
}

Jiwon Choi ${ }^{1 \ddagger}$, Soohyun Lee ${ }^{1 \ddagger}$, Jongwon Choe ${ }^{2}$, Yura Chung ${ }^{1}$, Yae Eun Lee ${ }^{1}$, Jisu Kim², Myungwoong Kim*2, and Keewook Paeng*1

${ }^{1}$ Department of Chemistry, Sungkyunkwan University, Suwon 16419, Republic of Korea

${ }^{2}$ Department of Chemistry and Chemical Engineering, Inha University, Incheon 22212, Republic of Korea.

*Keewook Paeng: e-mail: paeng@skku.edu

*Myungwoong Kim: e-mail: mkim233@inha.ac.kr

${ }^{\ddagger}$ Equal contribution 


\section{Synthesis of polymer tethered probes}

\section{Chemical characterizations}

${ }^{1} \mathrm{H}$ NMR spectra were recorded in $\mathrm{CDCl}_{3}$ using JEOL JNM-ECZ400S $400 \mathrm{MHz}$ spectrometer or Ascend ${ }^{\mathrm{TM}} 500$ $\mathrm{MHz}$ spectrometer with the residual solvent peak $\left(\mathrm{CDCl}_{3}, 7.26 \mathrm{ppm}\right)$ as an internal reference. High resolution mass spectroscopy was conducted with JMS900 mass spectrometer using FAB method at the Korea Basic Institute (Daegu).

\subsection{Amine-functionalized PDI}

\section{One-end amine-functionalized PDI}

Synthesis of $\mathrm{N}$-hexylamino- $N^{\prime}$-pentyl-3,4,9,10-perylenedicarboxylic diimide (5-PDI-6- $\left.\mathrm{NH}_{2}, \quad 4\right)$ and $\mathrm{N}$ dodecylamino- $N$ '-pentyl-3,4,9,10-perylenedicarboxylic diimide (6-PDI-12- $\mathrm{NH}_{2}$, 5). (The numbers in nomenclature of materials indicate length of alkyl chain.)

3,4,9,10-perylenetetracarboxylic dianhydride (PTCDA, 98\%, $1.00 \mathrm{~g}, 2.50 \mathrm{mmol}$, Alfa Aesar), potassium hydroxide (KOH, 95\%, 0.443 g, 7.49 mmol, Samchun Chemicals), and deionized (DI) water (50 mL) were mixed and stirred at $90{ }^{\circ} \mathrm{C}$ for $4 \mathrm{hrs}$. Then, $\mathrm{pH}$ of the mixture was adjusted to 6.0 by adding aqueous phosphoric acid $\left(\mathrm{H}_{3} \mathrm{PO}_{4}, 85 \mathrm{wt} \%\right.$, Sigma-Aldrich) solution (10 vol\%). The mixture was further stirred at $90{ }^{\circ} \mathrm{C}$ for $4 \mathrm{hrs}$. A solid $(3,4,9,10-$ perylenetetracarboxylic acid monoanhydride monopotassium carboxylate) was collected by filtration and dried under a vacuum. The solid was then mixed with 1-pentylamine $\left(\mathrm{C}_{5} \mathrm{H}_{13} \mathrm{~N}, 98 \%\right.$, 4.4 equiv, Alfa Aesar.) in DI water $(50 \mathrm{~mL})$ and stirred for $4 \mathrm{hrs}$ at room temperature and $90{ }^{\circ} \mathrm{C}$ for $2 \mathrm{hrs}$. The mixture was acidified with aqueous hydrogen chloride ( $\mathrm{HCl}, 35 \mathrm{wt} \%$, Matsunoen Chemicals) solution (10 vol\%) and the precipitate was filtered, washed with ethanol $\left(\mathrm{C}_{2} \mathrm{H}_{5} \mathrm{OH}, 99.9 \%\right.$, Samchun Chemicals $)$ and DI water to remove residual amine, and dried under vacuum. The solid was added to aqueous $\mathrm{KOH}$ solution $(10 \mathrm{wt} \%)$, then stirred at $90{ }^{\circ} \mathrm{C}$. An aqueous potassium chloride ( $\mathrm{KCl}, 99 \%$, Samchun Chemicals) solution ( $8 \mathrm{wt} \%$ ) was then slowly added to selectively solidify N-pentyl3,4,9,10-perylenetetracarboxylic-3,4-anhydride-9,10-imide (3). ${ }^{1-2}$ Collected solid was added to DI water, followed by stirring at room temperature. Undissolved solid was removed by filtration, then the solution was acidified by a slow addition of aqueous hydrogen chloride $(\mathrm{HCl})$ solution $(20 \mathrm{vol} \%)$, resulting in the precipitation of compound 3. The solid was collected by filtration and washed with DI water, followed by drying in a vacuum oven. Compound 3 was mixed with 1,6-diaminohexane $\left(\mathrm{C}_{6} \mathrm{H}_{16} \mathrm{~N}_{2},>98 \%, 20\right.$ equiv., for 5-PDI-6- $\mathrm{NH}_{2}$, Alfa Aesar) or 1,12diaminododecane $\left(\mathrm{C}_{12} \mathrm{H}_{28} \mathrm{~N}_{2},>98 \%\right.$, TCI Chemicals) (20 equiv., for 5-PDI-12- $\left.\mathrm{NH}_{2}\right)$, and DI water (100 mL), then stirred for $3 \mathrm{hrs}$ at $60{ }^{\circ} \mathrm{C}$. The precipitate was collected by filtration and washed with ethanol and DI water, followed by drying under vacuum. (Scheme S1) ${ }^{1} \mathrm{H}$ NMR (5-PDI-6-NH $\left.2, \mathrm{CDCl}_{3}\right) \delta=8.69(\mathrm{~d}, 4 \mathrm{H}, \mathrm{Ar}-\mathrm{H}), 8.62(\mathrm{~d}, 4 \mathrm{H}, \mathrm{Ar}-\mathrm{H})$, $4.21\left(\mathrm{td}, 4 \mathrm{H}, \alpha-\mathrm{CH}_{2}\right), 2.70\left(\mathrm{t}, 2 \mathrm{H}, \mathrm{NH}_{2}-\mathrm{CH}_{2}\right), 0.94\left(\mathrm{t}, 3 \mathrm{H}, \mathrm{CH}_{3}\right) \mathrm{ppm} .{ }^{1} \mathrm{H}$ NMR $\left(5-\mathrm{PDI}-12-\mathrm{NH}_{2}, \mathrm{CDCl}_{3}\right) \delta=8.70(\mathrm{~d}$, 
4H, Ar-H), 8.64 (d, 4H, Ar-H), 4.21 (td, 4H, $\left.\alpha-\mathrm{CH}_{2}\right), 2.67$ (t, 2H, NH$\left.-\mathrm{CH}_{2}\right), 0.87$ (t, 3H, $\left.\mathrm{CH}_{3}\right)$ ppm.
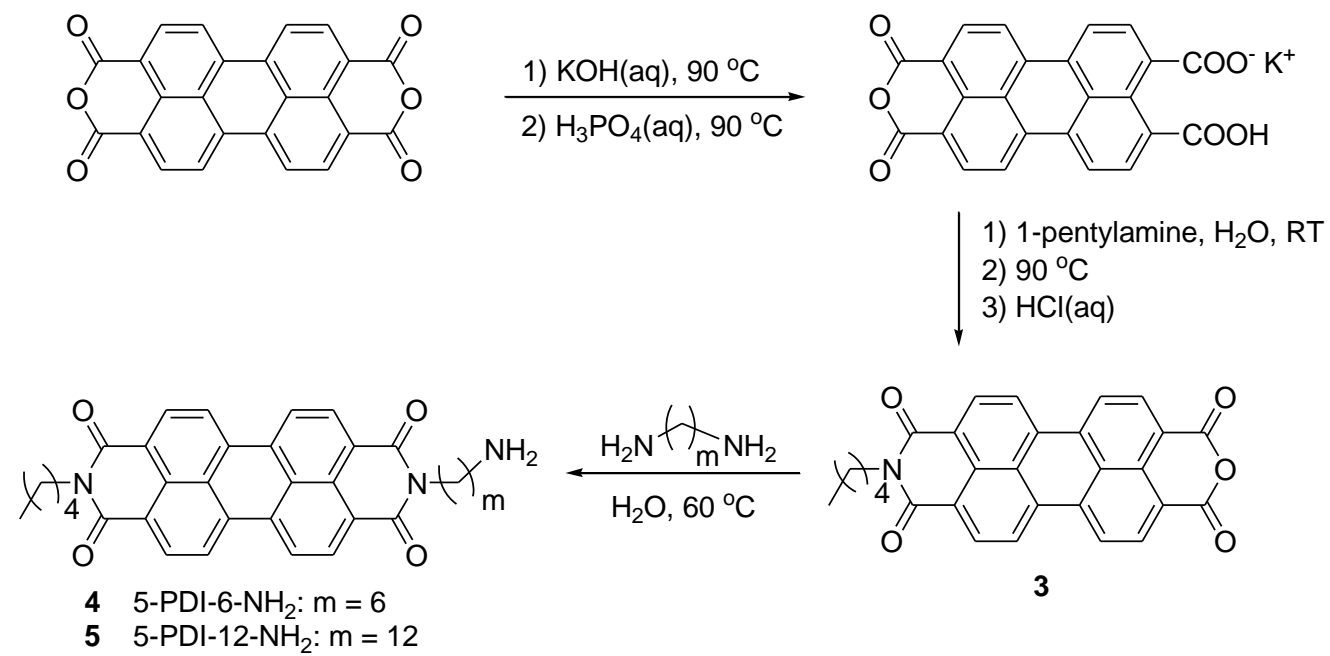

3

Scheme S1. Synthesis of primary amine functionalized asymmetric perylene probes (5-PDI-6-NH ${ }_{2}$ and 5-PDI-12$\mathrm{NH}_{2}$ ).

\section{Both-ends amine-functionalized PDI}

Synthesis of $\mathrm{N}, \mathrm{N}^{\prime}$-bis(hexylamino)-3,4,9,10-perylenedicarboxylic diimide ( $\left.\mathrm{NH}_{2}-6-\mathrm{PDI}-6-\mathrm{NH}_{2}, 6\right)$ and $\mathrm{N}, \mathrm{N}^{\prime}$ bis(dodecylamino)-3,4,9,10-perylenedicarboxylic diimide $\left(\mathrm{NH}_{2}-12-\mathrm{PDI}-12-\mathrm{NH}_{2}, 7\right)$. (The numbers in nomenclature of materials indicate length of alkyl chain.)

3,4,9,10-perylenetetracarboxylic dianhydride (1.00 g, $2.50 \mathrm{mmol})$ was mixed with 1,6-diaminohexane $(7.05 \mathrm{~mL}$, 50.0 mmol, for $\mathrm{NH}_{2}$-6-PDI-6- $\mathrm{NH}_{2}$ ) or 1,12-diaminododecane (10.22 g, $50.0 \mathrm{mmol}$, for $\left.\mathrm{NH}_{2}-12-\mathrm{PDI}-12-\mathrm{NH}_{2}\right)$ in DI water $(100 \mathrm{~mL})$. The mixture was stirred at $60{ }^{\circ} \mathrm{C}$ for $3 \mathrm{hrs}$, then the solid was collected by filtration and washed with ethanol and water. The product was then dried under vacuum. (Scheme S2) ${ }^{1} \mathrm{H}$ NMR $\left(\mathrm{NH}_{2}-6-\mathrm{PDI}-6-\mathrm{NH}_{2}\right.$, $\left.\mathrm{CDCl}_{3}\right) \delta=8.72(\mathrm{~d}, 4 \mathrm{H}), 8.66(\mathrm{~d}, 4 \mathrm{H}), 5.35(\mathrm{~s}, 4 \mathrm{H}), 4.22(\mathrm{~m}, 4 \mathrm{H}), 2.69(\mathrm{~m}, 4 \mathrm{H}), 1.8-0.8$ (alkyl chain) ppm. ${ }^{1} \mathrm{H}$ NMR $\left(\mathrm{NH}_{2}-12-\mathrm{PDI}-12-\mathrm{NH}_{2}, \mathrm{CDCl}_{3}\right) \delta=8.72(\mathrm{~d}, 4 \mathrm{H}), 8.66(\mathrm{~d}, 4 \mathrm{H}), 5.34(\mathrm{~s}, 4 \mathrm{H}), 4.20(\mathrm{~m}, 4 \mathrm{H}), 2.67(\mathrm{~m}, 4 \mathrm{H}), 1.8-0.8$ (alkyl chain) ppm.
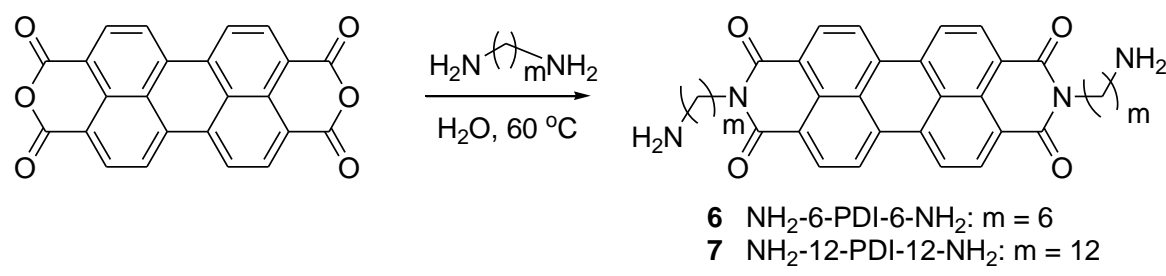

Scheme S2. Synthesis of primary amine functionalized symmetric perylene probes $\left(\mathrm{NH}_{2}-6-\mathrm{PDI}-6-\mathrm{NH}_{2}\right.$ and $\mathrm{NH}_{2}-$ 12-PDI-12- $\mathrm{NH}_{2}$ ). 


\subsection{Probe-tethered ATRP initiators}

General synthesis of probe-tethered ATRP initiators. (The numbers in nomenclature of materials indicate length of alkyl chain.)

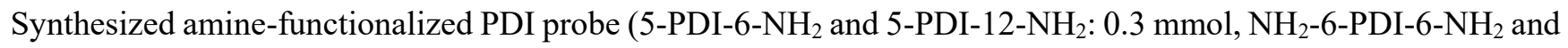
$\mathrm{NH}_{2}$-12-PDI-12- $\mathrm{NH}_{2}: 0.8 \mathrm{mmol}$ ) was dissolved in $120 \mathrm{~mL} \mathrm{CHCl}_{3}$ in a round-bottom flask with a stirring bar. An excess amount of triethylamine (TEA, $>99 \%$, Sigma-Aldrich) $(6.0 \mathrm{mmol}$ for 5-PDI-6-NH 2 and 5-PDI-12-NH $2,8.0$ mmol for $\mathrm{NH}_{2}-6-\mathrm{PDI}-6-\mathrm{NH}_{2}$ and $\mathrm{NH}_{2}-12-\mathrm{PDI}-12-\mathrm{NH}_{2}$ ) was added to the solution, then purged with nitrogen. $\alpha$ bromoisobutyryl bromide (BiBB, $9.0 \mathrm{mmol}$ for 5-PDI-6- $\mathrm{NH}_{2}$ and 5-PDI-12- $\mathrm{NH}_{2}, 8.0 \mathrm{mmol}$ for $\mathrm{NH}_{2}-6-\mathrm{PDI}-6-\mathrm{NH}_{2}$ and $\mathrm{NH}_{2}-12-\mathrm{PDI}-12-\mathrm{NH}_{2}$ ) was injected into the flask. The mixture was stirred at room temperature for overnight, then the mixture was filtered to remove insoluble residues in the solution. The solution was washed with DI water, then the organic layer was dried over anhydrous sodium sulfate $\left(\mathrm{Na}_{2} \mathrm{SO}_{4},>98 \%\right.$, Samchun Chemicals). The solvent was removed by rotary evaporation, followed by drying further in a vacuum oven at room temperature for $24 \mathrm{hrs}$. (Scheme S3) ${ }^{1} \mathrm{H}$ NMR (5-PDI-6-Br, $\left.\mathrm{CDCl}_{3}\right) \delta=8.48(\mathrm{~d}, 4 \mathrm{H}), 8.33(\mathrm{~d}, 4 \mathrm{H}), 4.17(\mathrm{t}, 4 \mathrm{H}), 3.28(\mathrm{~m}, 2 \mathrm{H}), 1.95(\mathrm{~s}, 6 \mathrm{H})$, 1.8-0.8 (alkyl chain) ppm; HRMS (FAB) m/z calcd. for $\mathrm{C}_{39} \mathrm{H}_{38} \mathrm{BrN}_{3} \mathrm{O}_{5}(\mathrm{M}+\mathrm{H})^{+}$708.2073; found, 708.2077. ${ }^{1} \mathrm{H}$ NMR (5-PDI-12-Br, $\left.\mathrm{CDCl}_{3}\right) \delta=8.69(\mathrm{~d}, 4 \mathrm{H}), 6.30(\mathrm{~d}, 4 \mathrm{H}), 4.21(\mathrm{t}, 4 \mathrm{H}), 3.24(\mathrm{~m}, 2 \mathrm{H}), 1.95$ (s, 6H), 1.8-0.8 (alkyl chain) ppm; HRMS (FAB) m/z calcd. for $\mathrm{C}_{45} \mathrm{H}_{50} \mathrm{BrN}_{3} \mathrm{O}_{5}(\mathrm{M}+\mathrm{H})^{+}$792.3012; found, 792.3007. ${ }^{1} \mathrm{H}$ NMR (Br-6-PDI6- $\left.\mathrm{Br}, \mathrm{CDCl}_{3}\right) \delta=8.70(\mathrm{~d}, 4 \mathrm{H}), 8.64(\mathrm{~d}, 4 \mathrm{H}), 4.22(\mathrm{t}, 4 \mathrm{H}), 3.27$ (m, 4H), 1.95 (s, 12H), 1.8-0.8 (backbone) ppm; HRMS (FAB) $\mathrm{m} / \mathrm{z}$ calcd. for $\mathrm{C}_{44} \mathrm{H}_{46} \mathrm{Br}_{2} \mathrm{~N}_{4} \mathrm{O}_{6}(\mathrm{M}+\mathrm{H})^{+}$885.1862; found, 885.1864. ${ }^{1} \mathrm{H}$ NMR (Br-12-PDI-12-Br, $\left.\mathrm{CDCl}_{3}\right) \delta=8.69(\mathrm{~d}, 4 \mathrm{H}), 8.62(\mathrm{~d}, 4 \mathrm{H}), 4.20(\mathrm{t}, 4 \mathrm{H}), 3.24(\mathrm{~m}, 4 \mathrm{H}), 1.95(\mathrm{~s}, 12 \mathrm{H}), 1.8-0.8$ (backbone) ppm; HRMS (FAB) $\mathrm{m} / \mathrm{z}$ calcd. for $\mathrm{C}_{56} \mathrm{H}_{70} \mathrm{Br}_{2} \mathrm{~N}_{4} \mathrm{O}_{6}(\mathrm{M}+\mathrm{H})^{+}$1053.3740; found, 1053.3745.

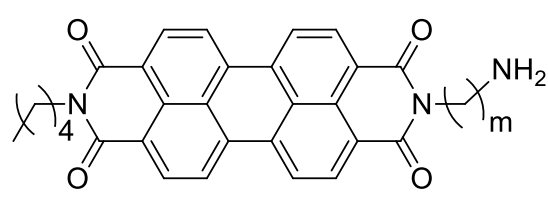

4 or 5

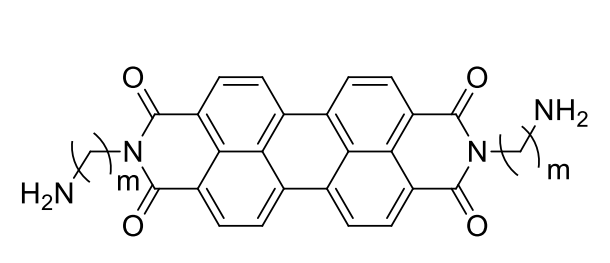

6 or 7
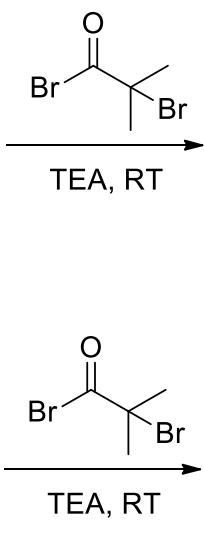

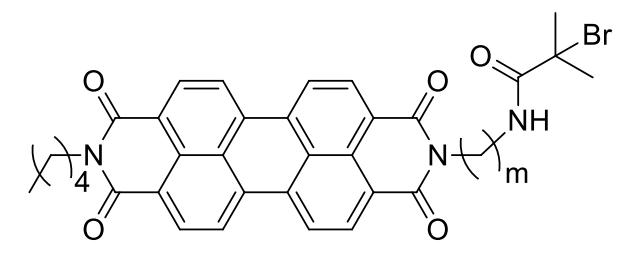

8 5-PDI-6-Br: $m=6$

9 5-PDI-12-Br: $m=12$

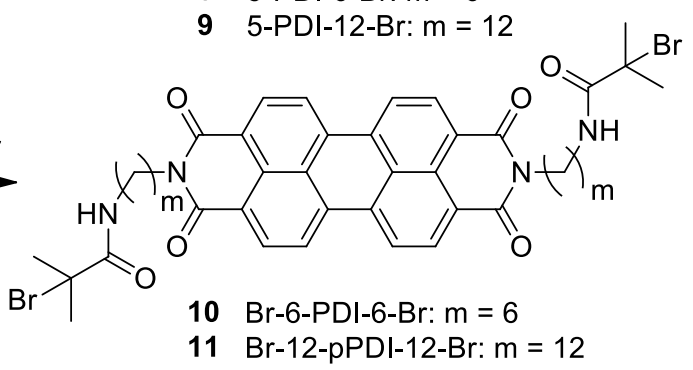

Scheme S3. Synthesis of PDI-functionalized ATRP initiators. 


\subsection{Atom transfer radical polymerization (ATRP) of MMA}

General procedure for ATRP of MMA from probe-tethered initiators. CuBr ( $>99 \%$, Sigma-Aldrich) (0.08 mmol), $\mathrm{CuBr}_{2}(>99 \%$, Acros Organics) (0.04 mmol), probe-tethered ATRP initiator (compound 8, 9, 10, or 11, $0.02 \mathrm{mmol}$ ), $\mathrm{N}, \mathrm{N}, \mathrm{N}^{\prime}, \mathrm{N}^{\prime \prime}, \mathrm{N}^{\prime \prime}-$ Pentamethyldiethylenetriamine (PMDETA, 99\%, Sigma-Aldrich) (0.08 mmol) and methyl methacrylate (MMA, $40.0 \mathrm{mmol}$ ) were used in all polymerizations. All reagents and a synthesized initiator were added to $4 \mathrm{~mL}$ anisole (99\%, Acros Organics) in a $10 \mathrm{~mL}$ Schlenk flask with a stirring bar. The solution was degassed by three freeze-pump-thaw cycles, then the flask was placed at an oil bath preheated to $90{ }^{\circ} \mathrm{C}$. The polymerization was proceeded for desired time, then stopped by exposing the reaction mixture to air. Resulting mixture was diluted with tetrahydrofuran (THF, $>99 \%$, Samchun Chemicals) and passed through an alumina column to remove copper residue. The solution was added dropwise to an excess amount of methanol for precipitation. The solid was collected by filtration and dried under vacuum oven at room temperature for 24 hrs. (Scheme S4)
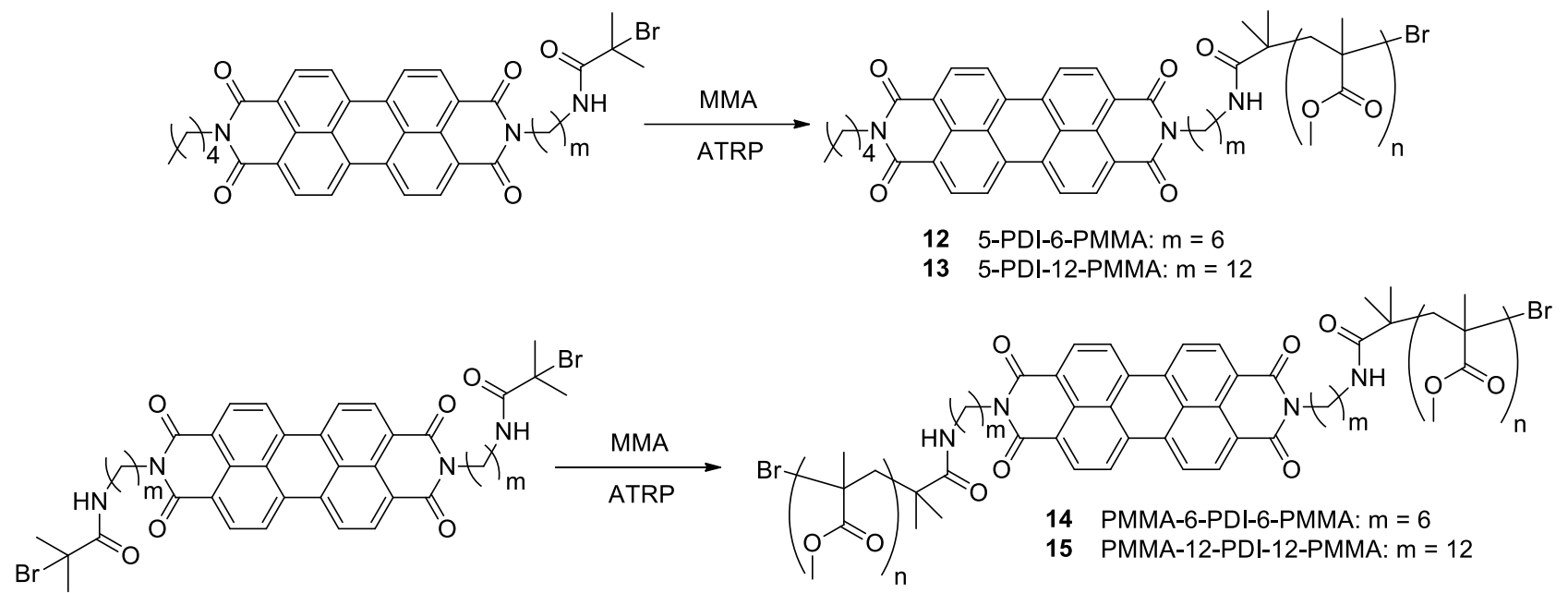

Scheme S4. Polymerization using PDI-functionalized ATRP initiators.

\subsection{Fractionation of as-synthesized probe-tethered PMMAs}

Possibly due to poor solubility of probe-tethered ATRP initiators in anisole, as-synthesized probe-tethered PMMAs $\left(\mathrm{M}_{\mathrm{w}}>100 \mathrm{~kg} / \mathrm{mol}\right)$ show relatively broad dispersity $(\fallingdotseq=1.4-1.8)$ at high conversion $(>40 \%)$ with unreacted probe-tethered initiators remaining in the polymerized samples. Unreacted probe-tethered initiators could obscure the interpretation of rotational dynamics of fluorescent probes covalently attached to the polymer chains. We effectively addressed these issues simultaneously to achieve samples with narrower dispersity $(\nexists<1.5)$ and also removing untethered free probes by fractionating as-polymerized samples and taking a fraction in the middle. This procedure automatically narrows the dispersity as well as removes untethered probe initiators from the polymer 
samples (Table S1). Fractioning of the sample was performed by gradually adding non-solvent to the as-synthesized probe-tethered PMMA/toluene solution and collecting precipitated sample by filtration when appropriate amount of precipitation was formed. ${ }^{3-4}$ In detail, when $\approx 4 \mathrm{~mL}$ of $n$-hexane was added to $15 \mathrm{~mL}$ of as-polymerized polymer solution $\left(\approx 6.7 \times 10^{-3} \mathrm{~g} / \mathrm{mL}\right)$, a higher molecular weight polymer fraction precipitate first which was collected by filtration. Decanted solution was then treated with an additional $\approx 4 \mathrm{~mL}$ of $n$-hexane which led to a second precipitated fraction of a lesser molecular weight polymers compared to the first fraction. By repeating this process for three more times, five different fractionated polymer samples were obtained. The precipitated samples were collected and dried at $100{ }^{\circ} \mathrm{C}$ under vacuum for $12 \mathrm{hrs}$, then were subjected to SEC analysis to measure molecular weight and dispersity $(\nexists)$ (Table S1), as well as to confirm the complete removal of unreacted probe initiators. SEC (Agilent Technologies, 1260 Infinity) equipped with both refractive index (RI) and UV-vis absorption detectors was used to for these purposes. SEC used three columns (PLgel MIXED-C, PLgel MIXED-C, and PLgel MIXED-C, Waters) which was calibrated by polystyrene standard samples $(0.162-6,570 \mathrm{~kg} / \mathrm{mol}$, Agilent).

\begin{tabular}{|c|c|c|c|c|}
\hline \multirow{2}{*}{ Sample } & \multicolumn{2}{|c|}{ As-synthesized } & \multicolumn{2}{c|}{ Fractionated } \\
\cline { 2 - 5 } & $\mathbf{M}_{\mathbf{w}}(\mathbf{g} / \mathbf{m o l})$ & $\boldsymbol{D}$ & $\mathbf{M}_{\mathbf{w}}(\mathbf{g} / \mathbf{m o l})$ & $\boldsymbol{\Xi}$ \\
\hline PMMA & 182,700 & 1.21 & & \\
\hline 5-PDI-12-PMMA & 265,200 & 1.79 & 211,100 & 1.30 \\
\hline 5-PDI-6-PMMA & 310,300 & 1.73 & 173,300 & 1.33 \\
\hline PMMA-12-PDI-12-PMMA & 97,500 & 1.38 & 75,000 & 1.31 \\
\hline PMMA-6-PDI-6-PMMA & 328,200 & 1.70 & 185,500 & 1.45 \\
\hline
\end{tabular}

Table S1. Molecular weight information of synthesized PMMA samples. (The numbers in the nomenclature of materials indicate the length of alkyl chains.)

Figure S1a compares UV-vis absorption signals of SEC excited at $521 \mathrm{~nm}$ where PDI probes have absorption $\lambda_{\max }$ in THF for as-polymerized and fractionated samples. The width of distribution decreased after the fractionation process and there was no peak found at the lower molecular weight region $(\approx 25.3 \mathrm{~mL}$ elution volume) indicating unreacted probe initiators were removed by the fractionation process. Figure S1b and S1c compares both RI and UV-vis absorption signals for the 1:1 mole ratio mixture of free probe, 5-PDI-5, with PMMA polymer and probetethered PMMA. As seen in Figure S1c, fractionated probe-tethered polymer sample does not show UV-vis absorption peak where the free probe show absorption (Figure S1b) but rather show peak at the same elution volume where RI detector shows the peak. This indicates that there is no untethered probe in the sample and all the probes are tethered to the polymer. The shift (tailing of the peak) at the later elution volume for the UV-vis absorption compared to RI is caused by the detection sensitivity difference between RI and UV-vis absorption detections where the former is sensitive to the weight and the latter to the number. ${ }^{5}$ 


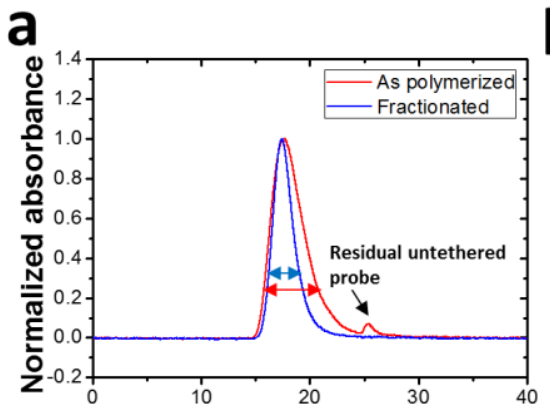

Elution Volume $(\mathrm{mL})$
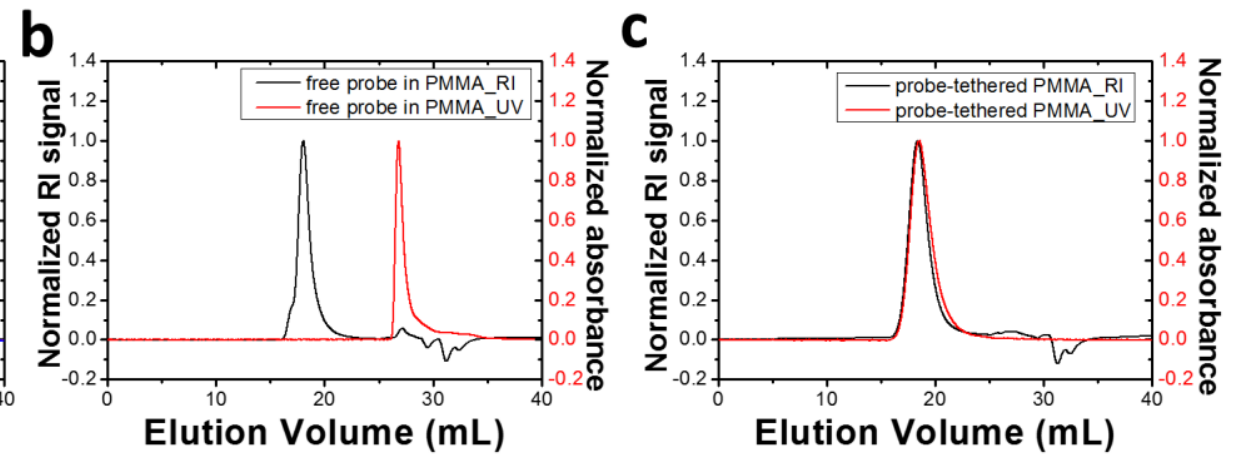

Figure S1. SEC chromatograms of (a) as polymerized and fractionated probe-tethered polymer traced by the UVvis detector, (b) free probe in PMMA, and (c) fractionated probe-tethered PMMA. (RI and UV-vis signals are black and red traces, respectively).

\subsection{Thin film preparation}

For free probes in polymer/toluene solution with a mixture of $4.04 \mathrm{wt} \%$ of PMMA and $\approx 10^{-5} \mathrm{M}$ concentration of free probe (1:100 dilution of fluorescent probe solution to polymer solution) was spin-coated at $2000 \mathrm{rpm}$ onto a piranha solution $\left(\mathrm{H}_{2} \mathrm{O}_{2}: \mathrm{H}_{2} \mathrm{SO}_{4}=1: 3\right)$ cleaned silicon wafer resulting in $\approx 200 \mathrm{~nm}$ polymer film which was confirmed by ellipsometry (Nanoview, SE MG 1000). For probe-tethered PMMAs, $4.04 \mathrm{wt} \%$ toluene solution which is a $0.05 \%$ mixture of probe-tethered and untethered polymer was spin-coated with the same procedure as the free probes in polymer samples. In both cases, probe concentration in the polymer film was estimated as $\approx 10^{-6} \mathrm{M}$. The spin-coated samples were subjected to thermal annealing at $417.5 \mathrm{~K}\left(\mathrm{~T}_{\mathrm{g}}+25 \mathrm{~K}\right.$ of PMMA) for $12 \mathrm{hrs}$ under vacuum $(\approx 10 \mathrm{mtorr})$ to remove residual solvent from the film and stress upon spin-coating.

\section{Photophysical properties of tethered probes}

Photophysical properties of tethered probes were characterized by UV-vis absorption and fluorescence spectra in toluene. Absorption spectra were recorded at room temperature using UV-vis absorption spectrometer (Mecasys, Optizen POP) with a resolution of $1 \mathrm{~nm}$ bandwidth between 400 and $700 \mathrm{~nm}$. Fluorescence spectra were acquired using fluorescence spectrometer (SCINCO, FS-2) in the same range with the excitation wavelength of $526 \mathrm{~nm}$ (absorption $\lambda_{\max }$ in toluene) and a resolution of $0.1 \mathrm{~nm}$. For both excitation and emission slits were set to $5 \mathrm{~nm}$. 


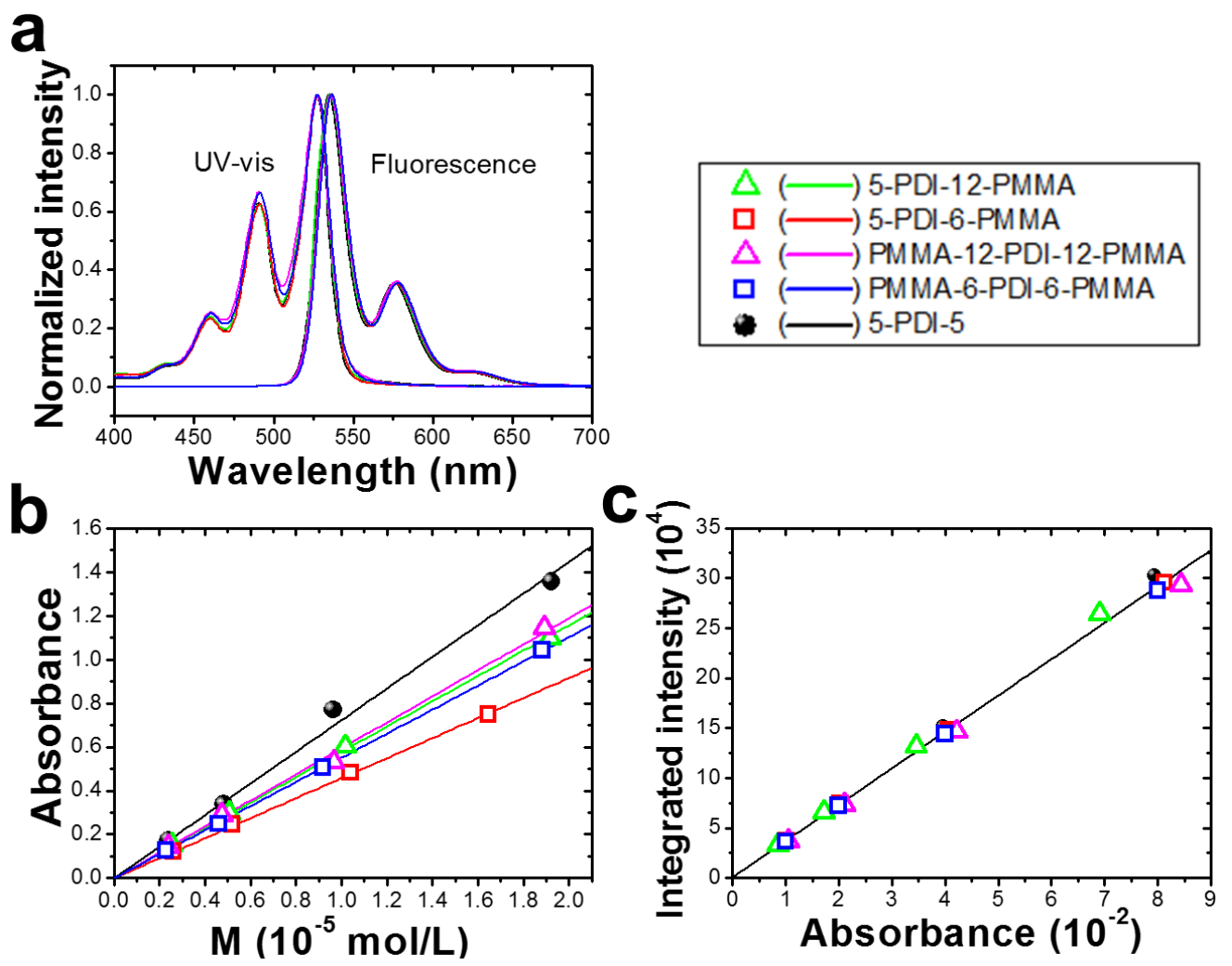

Figure S2. Plots showing (a) normalized absorbance and fluorescence spectra of 5-PDI-5 in PMMA/toluene solution and tethered probes in toluene, (b) absorbance as a function of probe concentration, (c) integrated fluorescence intensities as a function of absorbance.

Figure S2 shows absorption and emission spectrum of tethered probes in toluene solution which were compared to those of free probe of 5-PDI-5. It is shown that both absorption and emission spectrum for both one-end and both-ends tethered probes were not altered by tethering showing absorption and emission maxima at $526 \mathrm{~nm}$ and 534 nm, respectively. Concentrations of probe-tethered PMMAs in Figure S2 are actual molar concentrations of the PMMA and, in turn probe, which were estimated using molecular weight distributions of the PMMAs, obtained from SEC analysis. The molar extinction coefficient of the samples was obtained from the linear plots of their absorbance at $\lambda_{\max }$ versus concentration. Extinction coefficients of probes in all samples were found to be in the range of $4.6-6.0 \times 10^{4} \mathrm{~cm}^{-1} \mathrm{M}^{-1}$, which is less than the value of 5-PDI-5 $\left(7-8 \times 10^{4} \mathrm{~cm}^{-1} \mathrm{M}^{-1}\right)$ and the literature values of PDI probes. ${ }^{6-7}$ This result also confirms that there are no more than a single probe is attached to a polymer chain and there are no unreacted probe initiators left in the sample. Quantum yields were deduced by a relative method using Equation S1 and 5-PDI-5 probe as a reference standard. Quantum yields of the tethered probes are all close to unity $(\approx 0.97)$ determined by examining the slopes of integrated fluorescence intensities versus absorbance plot (Figure S2c), which also matched the quantum yield of the reported free probes. ${ }^{6-8}$ 


$$
\Phi_{x}=\Phi_{s t} \cdot\left(\frac{m_{x}}{m_{s t}}\right) \cdot\left(\frac{n_{x}}{n_{s t}}\right)^{2}
$$

Here, $\mathrm{m}_{\mathrm{x}}$ and $\mathrm{m}_{\mathrm{st}}$ are the slopes of integrated fluorescent intensity vs. absorbance plot for unknown and the standard, respectively, and $n_{x}$ and $n_{s t}$ are the refractive indices of the measured solvents which ratio was set to a unity as the same concentration of PMMA solution was used as a solvent in the current analysis.

\section{Fluorescent probe molecular rotation measurements}

\section{Optical measurements and data analysis}

Rotational dynamics of polymer-tethered probe in a host matrix was studied through imaging rotational fluorescent correlation microscopy (irFCM) as described in reference 9. In brief, excitation light from a continuous wave 532 nm laser (CNI, MGL-III-532) was delivered through a multimode fiber which was shaken mechanically to obtain randomly polarized and homogenously illuminated field of view. A set of half and quarter wave plates was placed prior to the multimode fiber to fine-control the magnitude of $\mathrm{s}$ and $\mathrm{p}$ polarized intensities in the randomly polarized light. The excited light was collimated and reflected by a dichroic mirror (Semrock, LPD02-532RU-25) which was then focused to the back of the objective lens (Olympus, LUCPLFLN, 60x, NA $=0.70, \mathrm{WD}=1.5-2.2 \mathrm{~mm}$ ), resulting in a field of view of $\approx 100 \mu \mathrm{m}$ in diameter. The fluorescence image was collected with the same objective lens and passed through the dichroic mirror followed by long-pass (Semrock, LP03-532RE-25) and band-pass (Semrock, FF01-571/72-25) filters to remove residual excitation light and optical noises. A Wollaston prism (Karl Lambrecht Corporation, MW2S-12-5) split the image into two orthogonal polarizations that were imaged onto an EMCCD camera (Andor iXon Ultra 897). The time-sequenced intensities of orthogonal polarization $\left(I_{s}(t), I_{p}(t)\right)$ were extracted from these images and the linear dichroism $\left(\operatorname{LD}(t)=\left[I_{s}(t)-I_{p}(t)\right] /\left[I_{s}(t)+I_{p}(t)\right]\right.$ was calculated. An autocorrelation of $\mathrm{LD}(\mathrm{t})$ was constructed via $\left[\Sigma \mathrm{t}^{\prime} \mathrm{a}\left(\mathrm{t}^{\prime}\right) \cdot \mathrm{a}\left(\mathrm{t}^{\prime}+\mathrm{t}\right)\right] /\left[\Sigma \mathrm{t}^{\prime} \mathrm{a}\left(\mathrm{t}^{\prime}\right) \cdot \mathrm{a}\left(\mathrm{t}^{\prime}\right)\right]$ where $\mathrm{a}(\mathrm{t})=\mathrm{LD}(\mathrm{t})-<\mathrm{LD}(\mathrm{t})>$. Typically, 100 regions of $5 \times 5$ pixels were analyzed and produced 100 autocorrelation decays in a single measurement, which were averaged to give one autocorrelation decay. Obtained averaged correlation decay was fitted to a stretched exponential function, $\mathrm{C}(\mathrm{t})=\mathrm{C}(0) \cdot \exp \left[-\left(\mathrm{t} / \tau_{\mathrm{fit}}\right)^{\beta}\right]$ which resulted in two fitting variables of a time

scale, $\tau_{\text {fit }}$, and a stretching exponent, $\beta$. The rotational relaxation time $\tau_{\mathrm{c}}$ was calculated from the values of $\tau_{\mathrm{fit}}$ and $\beta$ via $\tau_{\mathrm{c}}=\left(\tau_{\mathrm{fit}} / \beta\right) \cdot \Gamma(1 / \beta)$, where $\Gamma$ denotes the gamma function. The correlation function was fit until it decayed to 0.1. Data were analyzed using a custom program written in IDL (ITT Visual Information Solutions) software. ${ }^{9}$

\section{Normalized autocorrelation decays for free and tethered probes in PMMA.}

Figure S3 shows the normalized autocorrelation decays for two free probes (5-PDI-5, tbPDI) and four tethered 
probes in PMMA matrix at different temperatures indicated. The trajectory length and frame rate of the measurements were set to $\approx 400 \tau_{\text {fit }}$ and $15-20$ frames $/ \tau_{\mathrm{fit}}$, respectively. ${ }^{9}$ For all probes, whether they are tethered or not, autocorrelation decays faster at higher temperatures indicating probes rotating faster at elevated temperatures. The curved lines are fits to the stretched exponential functions for each data at different temperatures and fit results are presented in Figure 2 in main text and Figure S4.
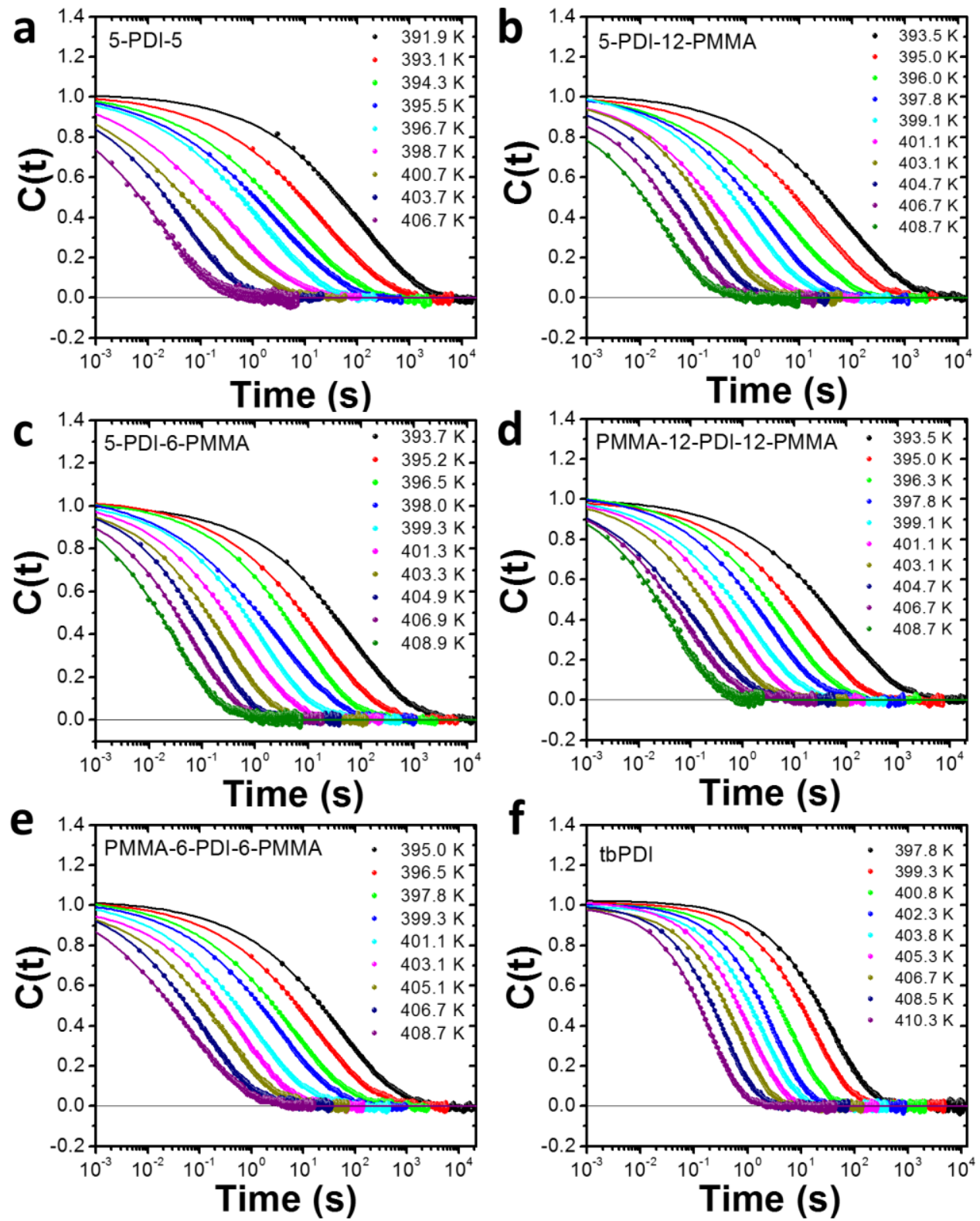

Figure S3. Normalized autocorrelation decays of free and tethered probes in PMMA matrix at different temperatures indicated. Solid lines represent fitting results to the stretched exponentials. 


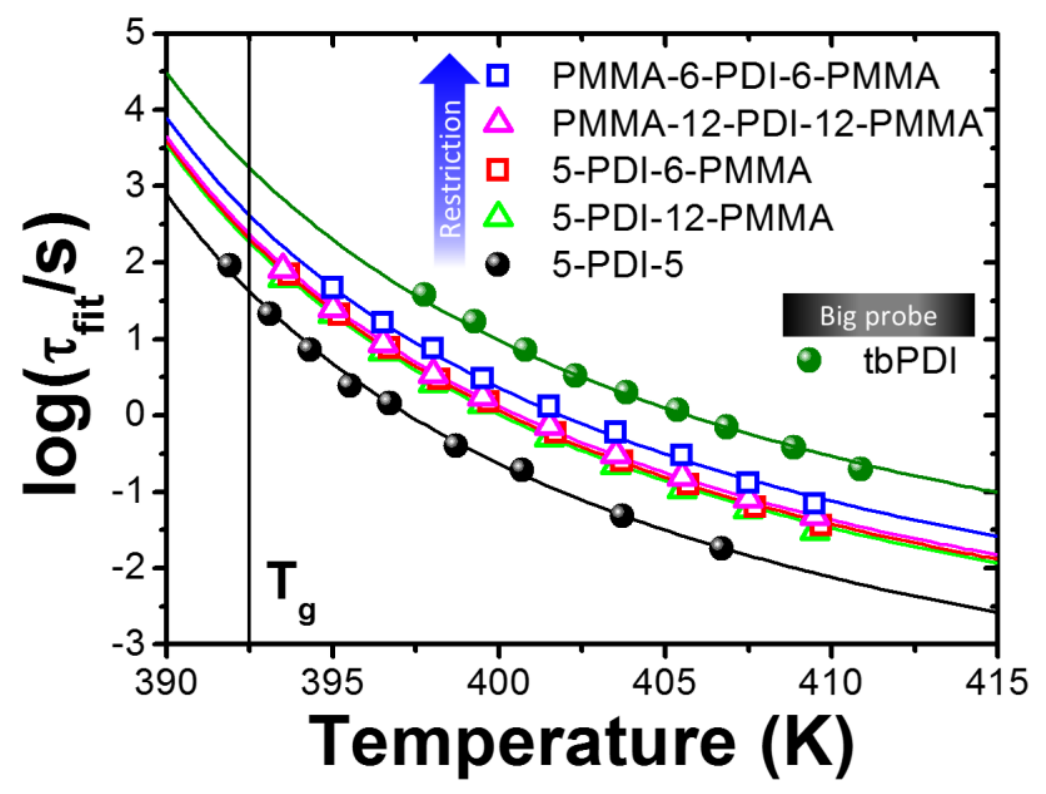

Figure S4. Temperature dependence of $\tau_{\text {fit }}$ for free probes and tethered probes in PMMA matrix. The vertical line indicates bulk $\mathrm{T}_{\mathrm{g}}$ value $\left(\mathrm{T}_{\mathrm{g}, \mathrm{DSC}}=392.5 \mathrm{~K}\right)$ of PMMA obtained using differential scanning calorimetry (DSC).

Figure $\mathbf{S} 4$ shows the temperature dependence of $\tau_{\text {fit }}$ for tethered-PDI probes and free tbPDI which were compared to free probe of 5-PDI-5. The lines in the figure are the VFT equation fit to the temperature dependence of $\tau_{\text {fit }}$ for 5-PDI-5 in PMMA and vertical shifts to match those of tethered probes. The fit values were $\log \left(\tau_{0}\right)=-5.76, \mathrm{~B}=$ 125.45 K, and $\mathrm{T}_{0}=375.50 \mathrm{~K}$ and the shifts are 0.64, 0.70, 0.75, and 1.00 decades for 5-PDI-12-PMMA, 5-PDI-6PMMA, PMMA-12-PDI-12-PMMA and PMMA-6-PDI-6-PMMA respectively which is in the order of increasing restriction to tethered probes by decreasing flexible alkyl chain linker length and increasing the tethering points from one-end to both-ends. A bigger probe of tbPDI have the sample temperature dependence with smaller 5-PDI5 with vertical shift of 1.60 decades. 


\section{References}

1. Nagao, Y.; Naito, T.; Abe, Y.; Misono, T., Synthesis and properties of long and branched alkyl chain substituted perylenetetracarboxylic monoanhydride monoimides. Dyes pigments 1996, 32 (2), 71-83.

2. Nagao, Y., Synthesis and properties of perylene pigments. Progress in organic coatings 1997, 31 (1-2), 43-49.

3. Jungnickel, J.; Weiss, F., Polymer fractionation by precipitation chromatography. Journal of Polymer Science 1961, 49 (152), 437-454.

4. $\quad$ Cantow, M. J. (Ed.), Polymer fractionation. Elsevier: 2013.

5. Karlsson, O., The composition of peat liquids: 1. Optimization of gpc parameters for the analysis of peat liquids. Fuel 1990, 69 (5), 608-612.

6. $\quad$ Chao, C.-C.; Leung, M.-k.; Su, Y. O.; Chiu, K.-Y.; Lin, T.-H.; Shieh, S.-J.; Lin, S.-C., Photophysical and electrochemical properties of 1, 7-diaryl-substituted perylene diimides. The Journal of organic chemistry 2005, 70 (11), 4323-4331.

7. $\quad$ Prathapan, S.; Yang, S. I.; Seth, J.; Miller, M. A.; Bocian, D. F.; Holten, D.; Lindsey, J. S., Synthesis and excited-state photodynamics of perylene- porphyrin dyads. 1. Parallel energy and charge transfer via a diphenylethyne linker. The Journal of Physical Chemistry B 2001, 105 (34), 8237-8248.

8. Huang, C.; Barlow, S.; Marder, S. R., Perylene-3, 4, 9, 10-tetracarboxylic acid diimides: synthesis, physical properties, and use in organic electronics. The Journal of organic chemistry 2011, 76 (8), 2386-2407.

9. Lee, S.; Choi, J.; Choe, J.; Kim, M.; Paeng, K., Segmental dynamics of polymer by rotational fluorescence correlation microscopy. The Journal of Chemical Physics 2018, 149 (16), 164910. 